\title{
Chemical clues on the formation of planetary systems
}

\author{
Elisa Delgado Mena ${ }^{1,2}$, Garik Israelian ${ }^{1,2}$, Jonay I. González \\ Hernández ${ }^{1,2}$, Jade C. Bond ${ }^{3}$, Nuno C. Santos ${ }^{4,5}$, Stéphane Udry ${ }^{6}$ and \\ Michel Mayor ${ }^{6}$ \\ ${ }^{1}$ Instituto de Astrofísica de Canarias, E-38200 La Laguna, Tenerife, Spain. email: edm@iac.es \\ ${ }^{2}$ Departamento de Astrofísica, Universidad de La Laguna, 38205 La Laguna, Tenerife, Spain. \\ ${ }^{3}$ Planetary Science Institute, 1700 E. Fort Lowell, Tucson, AZ 85719, USA. \\ ${ }^{4}$ Centro de Astrofísica, Universidade do Porto, Rua das Estrelas, 4150-762 Porto, Portugal. \\ ${ }^{5}$ Departamento de Física e Astronomia, Faculdade de Ciências, Universidade do Porto, \\ Portugal. \\ ${ }^{6}$ Observatoire de Genève, $51 \mathrm{ch}$. des Maillettes, CH-1290 Sauverny, Switzerland.
}

\begin{abstract}
Theoretical studies suggest that $\mathrm{C} / \mathrm{O}$ and $\mathrm{Mg} / \mathrm{Si}$ are the most important elemental ratios in determining the mineralogy of terrestrial planets. The $\mathrm{C} / \mathrm{O}$ ratio controls the distribution of $\mathrm{Si}$ among carbide and oxide species, while $\mathrm{Mg} / \mathrm{Si}$ gives information about the silicate mineralogy. We find mineralogical ratios quite different from those of the Sun, showing that there is a wide variety of planetary systems which are not similar to Solar System. Many of planetary host stars present a $\mathrm{Mg} / \mathrm{Si}$ value lower than 1, so their planets will have a high Si content to form species such as $\mathrm{MgSiO}_{3}$. This type of composition can have important implications for planetary processes like plate tectonics, atmospheric composition or volcanism. Moreover, the information given by these ratios can guide us in the search of stars more probable to form terrestrial planets.
\end{abstract}

Keywords. stars: abundances, stars: atmospheres, stars: fundamental parameters, planetary systems, planetary systems: formation

\section{Introduction}

The study of the photospheric stellar abundances of planet host stars is the key to understand how and which of the protoplanetary clouds form planets and which do not. These studies also help us to investigate the internal and atmospheric structure and composition of extrasolar planets.

One remarkable characteristic of planet host stars is that they are considerably metal rich when compared with single field dwarfs (Gonzalez 1998, Gonzalez et al. 2001, Santos et al. 2000, 2001, 2004, Fischer \& Valenti 2005). Two main explanations have been suggested to clarify this difference. The first of these is that the origin of this metallicity excess is primordial, so the more metals you have in the proto-planetary disk, the higher should be the probability of forming a planet. On the other hand, this excess might be produced by accretion of rocky material by the star some time after it reached the main-sequence. Recent studies on chemical abundances in stars with and without planets showed no important differences in $[\mathrm{X} / \mathrm{Fe}]$ vs. $[\mathrm{Fe} / \mathrm{H}]$ trends between both groups of stars (Takeda 2007, Bond et al. 2008, Neves et al. 2009, González Hernández et al. 2010). However, other works have reported less statistically significant enrichments in other species such as C, Na, Si, Ni, Ti, V, Co, Mg and Al (Santos et al. 2000, Gonzalez 
et al. 2001, Sadakane et al. 2002, Bodaghee et al. 2003, Fischer \& Valenti 2005, Beirão et al. 2005, Gilli et al. 2006, Bond et al. 2006, Gonzalez \& Laws 2007).

These results have important implications for models of giant planet formation and evolution. There are two major planet formation models: the core accretion model (Pollack et al. 1996), more likely to form planets in the inner disk, and the disk instability model (Boss 1997), which is in better agreement with the conditions in the extended disk. In the core accretion model, planet formation is dependent on the dust content of the disk (Pollack et al. 1996) while in the disk instability model it is not so clear (Boss 2002, Cai et al. 2006). Present observations are thus more compatible with core accretion model although they do not exclude disk instability.

Theoretical studies suggest that $\mathrm{C} / \mathrm{O}$ and $\mathrm{Mg} / \mathrm{Si}$ are the most important elemental ratios in determining the mineralogy of terrestrial planets and they can give us information about the composition of these planets. The $\mathrm{C} / \mathrm{O}$ ratio controls the distribution of $\mathrm{Si}$ among carbide and oxide species, while $\mathrm{Mg} / \mathrm{Si}$ gives information about the silicate mineralogy (Bond et al. 2010a, Bond et al. 2010b). Bond et al. (2010b) carried out simulations of planet formation where the chemical composition of the protoplanetary cloud was taken as an input parameter. Terrestrial planets were found to form in all the simulations with a wide variety of chemical compositions so these planets might be very different from the Earth. In order to investigate the mineralogical characteristics of those systems we will present $\mathrm{C} / \mathrm{O}$ and $\mathrm{Mg} / \mathrm{Si}$ ratios in a sample of 71 and 380 stars with and without detected planets, respectively, using new high quality spectra from the HARPS GTO sample (Mayor et al. 2003) and very precise stellar parameters (Sousa et al. 2008).In addition we use high quality spectroscopic observations for 42 stars hosting planets from the CORALIE survey, using the same spectral tools to determine their stellar parameters (Santos et al. 2004, 2005), and thus ensuring that the final sample is homogeneous.

\section{Abundances}

For all the elements we performed a standard LTE analysis with the 2002 revised version of the spectral synthesis code MOOG (Sneden 1973) and a grid of Kurucz ATLAS9 atmospheres with overshooting (Kurucz 1993), by measuring the equivalent width (EW) of the different lines with the ARES program† (Sousa et al. 2007).

$\mathrm{Mg}$ and Si abundances were calculated using the line list of Neves et al. (2009), adding a $\mathrm{Mg}$ line at $\lambda 6318.72 \AA$. On the other hand, $\mathrm{C}$ and $\mathrm{O}$ abundances were determined by measuring the equivalent widths of $C I$ lines at $\lambda 5380.3 \AA$ and $\lambda 5052.2 \AA$ and $O I$ forbidden line at $\lambda 6300 \AA$. We removed from the sample stars with $\mathrm{T}_{\text {eff }}<5100 \mathrm{~K}$ since $\mathrm{C}$ abundance is not reliable for those stars. In addition, the spectral region around the forbidden line has telluric lines which can be blended with the $O I$ line in some stars. So we made a detailed observation of the spectra to remove these objets from the sample in order to avoid wrong values of the $\mathrm{O}$ abundance. This, together with the limitation on $T_{\text {eff }}$, makes a final sample of 69 and 270 stars with and without detected planets from HARPS, and 31 stars with planets from other surveys.

In Fig. $1[\mathrm{X} / \mathrm{Fe}]$ ratios as a function of metallicity are plotted. There is an average overabundance in the total planet-host stars with respect to the comparison sample stars for all the elements. Since targets with planets are on average more metal-rich than the stars of comparison sample, their abundance distributions correspond to the extensions of the comparison sample trends at high metallicity. Such a trend supports the primordial

$\dagger$ The ARES code can be downloaded at http://www.astro.up.pt/ sousasag/ares/ 

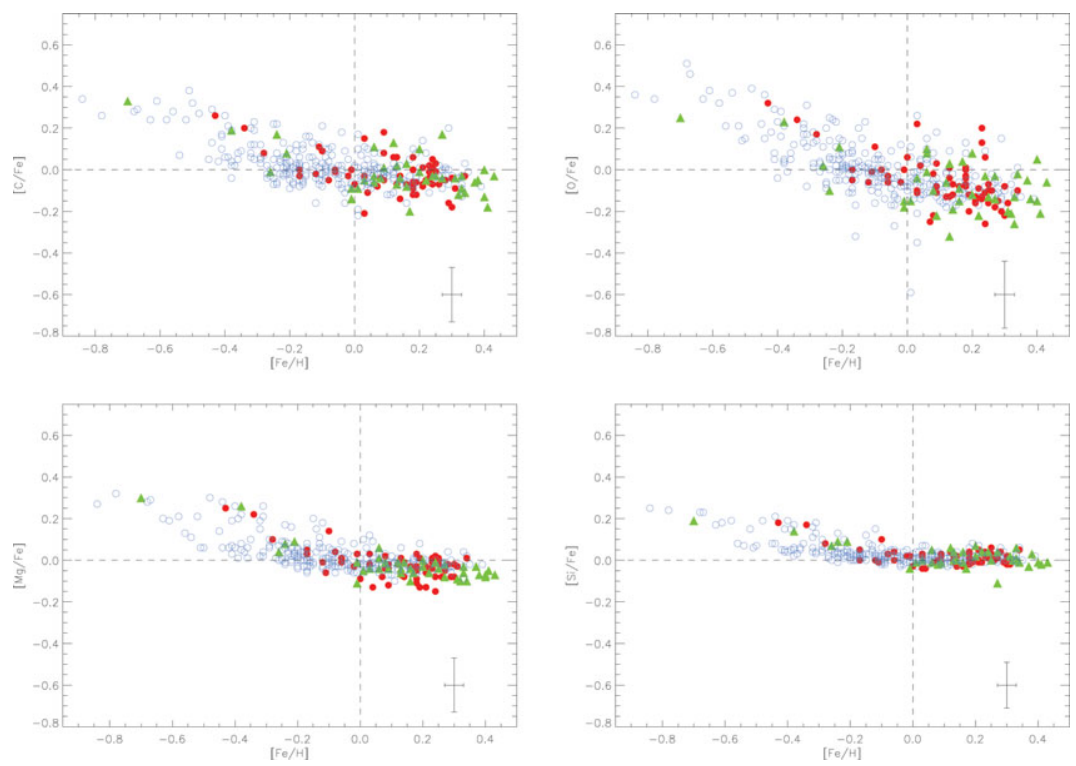

Figure 1. $[\mathrm{X} / \mathrm{Fe}]$ ratios as a function of metallicity for $\mathrm{C}, \mathrm{O}, \mathrm{Mg}$ and $\mathrm{Si}$.

scenario as an explanation of the overmetallicity of planet-host stars. The samples of stars both with and without detected planets behave quite similarly except for $\mathrm{Mg}$. At subsolar metallicities all stars present high $\mathrm{Mg}$ abundances irrespective of $T_{\text {eff }}$. However, this is not the case for $[\mathrm{Fe} / \mathrm{H}] \geqslant 0$, where stars without detected planets have higher $\mathrm{Mg}$ abundances. Nevertheless, this effect dissapears when we take into account only solar analogs, with $5600<T_{\text {eff }}<5950 \mathrm{~K}$, perhaps due to the low number of stars with planets in this group. Therefore, it might be an effect in $\mathrm{Mg}$ abundances due to the presence of planetary companions.

\section{3. $\mathrm{C} / \mathrm{O}$ vs $\mathrm{Mg} / \mathrm{Si}$}

In Fig. 2, $\mathrm{C} / \mathrm{O}$ ratios as a function of $\mathrm{Mg} / \mathrm{Si}$ are presented for different temperature ranges. These ratios are calculated as:

$$
\mathrm{A} / \mathrm{B}=N_{\mathrm{A}} / N_{\mathrm{B}}=10^{\log \epsilon(\mathrm{A})} / 10^{\log \epsilon(\mathrm{B})}
$$

where $\log \epsilon(\mathrm{A})$ and $\log \epsilon(\mathrm{B})$ are the absolute abundances, so they are not dependent on solar reference abundances.

In our sample, $34 \%$ of stars with known planets have $\mathrm{C} / \mathrm{O}$ values greater than 0.8 (see Table 1), which means that under the assumption of equilibrium those systems will contain carbide-rich phases (such as graphite, $\mathrm{SiC}$ and $\mathrm{TiC}$ ) in the innermost regions of the disk. Metallic Fe and $\mathrm{Mg}$-silicates such as olivine $\left(\mathrm{Mg}_{2} \mathrm{SiO}_{4}\right)$ and pyroxene $\left(\mathrm{MgSiO}_{3}\right)$ are also present and are located further from the host star. Terrestrial planets forming in these planetary systems are expected to be C-rich, containing significant amounts of $\mathrm{C}$ in addition to $\mathrm{Si}, \mathrm{Fe}, \mathrm{Mg}$ and $\mathrm{O}$. Those systems may possess an alternative mass distribution profile for solid material, potentially making it easier either for giant planets to form closer to the host star than previously expected or for terrestrial planets to form in the inner regions of the disk (Bond et al. 2010b). However, we find no evidence of any trends with $\mathrm{C} / \mathrm{O}$ values for either planetary period, semi-major axis or mass. As such, it appears that any effects of an alternative solid mass distribution due to high 
concentrations of refractory C-rich material are not preserved in the architecture of the system. This is believed to be due to the fact that Bond et al. (2010b) only considered equilibrium-driven condensation and did not include the effects of disequilibrium or the migration and radial mixing of material within the disk. Simulations addressing this issue are in progress. It should be noted, however, that we are still only able to detect giant planets. This conclusion may be not hold for extrasolar terrestrial planets which require significantly smaller amounts of solid material.

Table 1. $\mathrm{C} / \mathrm{O}$ and $\mathrm{Mg} / \mathrm{Si}$ distributions for stars with planets.

\begin{tabular}{ccl}
\hline Ratio & Percentage & Principal Composition \\
\hline \hline $\mathrm{C} / \mathrm{O}>0.8$ & $34 \%$ & $\begin{array}{l}\text { graphite, } \mathrm{TiC} \text { and solid } \mathrm{Si} \text { as } \mathrm{SiC} \\
\text { solid } \mathrm{Si} \text { as } \mathrm{SiO}_{4}{ }^{4-} \text { or } \mathrm{SiO}_{2}\end{array}$ \\
$\mathrm{C} / \mathrm{O}<0.8$ & $66 \%$ & \\
\hline $\mathrm{Mg} / \mathrm{Si}<1$ & $56 \%$ & $\begin{array}{l}\text { pyroxene, metallic } \mathrm{Fe} \text { and excess } \mathrm{Si} \text { as feldspars } \\
\text { equal pyroxene and olivine } \\
1<\mathrm{Mg} / \mathrm{Si}<2\end{array}$ \\
$\begin{array}{c}\mathrm{Mg} / \mathrm{Si}>2 \\
0 \%\end{array}$ & 0 olivine and excess $\mathrm{Mg}$ as $\mathrm{MgO}$ \\
\hline
\end{tabular}

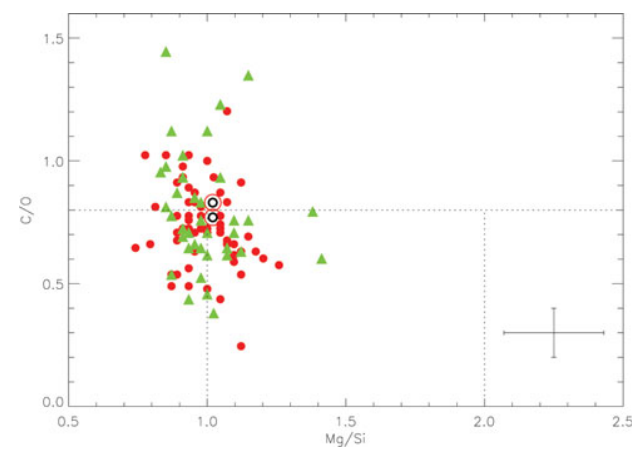

Figure 2. C/O vs Mg/Si for stars with planets from the HARPS GTO sample (red filled circles). Green triangles are stars with planets from other surveys. Solar abundances are calculated with the Kurucz Solar Atlas and the Harps daytime spectrum.

On the other hand, $66 \%$ of stars with known planets have $\mathrm{C} / \mathrm{O}$ values lower than 0.8 and $\mathrm{Si}$ will be present in the solid form as $\mathrm{SiO}_{4}{ }^{4-}$ or $\mathrm{SiO}_{2}$, predominantly forming $\mathrm{Mg}$ silicates. Silicate mineralogy will be controlled by $\mathrm{Mg} / \mathrm{Si}$ ratio. For systems with a Mg/Si value between 1 and 2, the silicates present are predominately olivine and pyroxene in a condensation sequence closely resembling Solar. This is expected to result in the production of terrestrial planets similar in composition to that of Earth (in that their composition will be dominated by $\mathrm{O}, \mathrm{Fe}, \mathrm{Mg}$ and $\mathrm{Si}$, with small amounts of $\mathrm{Ca}$ and $\mathrm{Al}$ also present). However, $56 \%$ of all planetary host stars in this study have a $\mathrm{Mg} / \mathrm{Si}$ value less than 1 . For such a composition, the solid component of the disk is dominated by approximately equal amounts of pyroxene and metallic Fe with minimal amounts of olivine present. Feldspars are also likely to be present as all available $\mathrm{Mg}$ is partioned into pyroxene, leaving excess $\mathrm{Si}$ available to form other silicate species.

Such an excess of Si content is predicted to produce a quartz-feldspar rich terrestrial planet with a composition more like that of Earth's continental crust material than 
that of Earth's olivine-dominated mantle. A composition such as this can have drastic implications for planetary processes such as plate tectonics and atmospheric composition. For example, volcanism on a Si-rich planet is expected to be intermediate to felsic in composition (i.e. $>52 \%$ silica by weight) due to the potentially high $\mathrm{SiO}_{2}$ content of the planet itself, producing igneous species such as andesite, rhyolite and granite. Eruptions may also be more explosive in nature due to the high viscosity of $\mathrm{SiO}_{2}$-rich magma trapping volatiles within the magma.

Although we also found stars very similar to our Sun, it is clear that a wide variety of planets will probably exist within extrasolar planetary systems. These results can give us hints of what type of terrestrial planets we could find in different stars and help to guide the future surveys of low-mass planets.

\section{References}

Beirão, P., Santos, N. C., Israelian, G., \& Mayor, M. 2005, Aछ A, 438, 251

Bodaghee, A., Santos, N. C., Israelian, G., \& Mayor, M. 2003, AESA, 404, 715

Bond, J. C., Tinney, C. G., Butler, R. P., Jones, H. R. A., Marcy, G. W., Penny, A. J., \& Carter, B. D. $2006, M N R A S, 370,163$

Bond, J. C., et al. 2008, ApJ, 682, 1234

Bond, J. C., Lauretta, D. S., \& O'Brien, D. P. 2010a, Icarus, 205, 321

Bond, J. C., O'Brien, D. P., \& Lauretta, D. S. 2010b, ApJ, 715, 1050

Boss, A. P. 1997, Science, 276, 1836

Boss, A. P. 2002, ApJ, 567, 149

Cai, K., Durisen, R. H., Michael, S., Boley, A. C., Mejía, A. C., Pickett, M. K., \& D’Alessio, P. 2006, ApJL, 636, L149

Gilli, G., Israelian, G., Ecuvillon, A., Santos, N. C., \& Mayor, M. 2006, A\&AA, 449, 723

Gonzalez, G. 1998, A\&A, 334, 221

Gonzalez, G., Laws, C., Tyagi, S., \& Reddy, B. E. 2001, AJ, 121, 432

Gonzalez, G. \& Laws, C. 2007, MNRAS, 378, 1141

González Hernández, J. I., Israelian, G., Santos, N. C., Sousa, S. G., Delgado Mena, E., Neves, V., \& Udry, S. 2010, ApJ, 720, 1592

Fischer, D. A. \& Valenti, J. 2005, AJ, 622, 1102

Kurucz, R. L. 1993, CD-ROMs, ATLAS9 Stellar Atmospheres Programs (Cambridge: Smithsonian Astrophys. Obs.)

Mayor, M. \& Queloz, D., et al.2003, The Messenger, 114,20

Neves, V., Santos, N. C., Sousa, S. G., Correia, A. C. M., \& Israelian, G. 2009, A\& A, 497, 563

Pollack, J. B., Hubickyj, O., Bodenheimer, P., Lissauer, J. J., Podolak, M., \& Greenzweig, Y. 1996, Icarus, 124, 62

Sadakane, K., Ohkubo, M., Takeda, Y., Sato, B. Kambe, E., \& Aoki, W. 2002, PASJ, 54, 911

Santos, N. C., Israelian, G., \& Mayor, M. 2000, $A \mathscr{E} A$, 363, 228

Santos, N. C., Israelian, G., \& Mayor, M. 2001, A\&A, 373, 1019

Santos, N. C., Israelian, G., \& Mayor, M. 2004, A\&A, 415, 1153

Santos, N. C., Israelian, G., Mayor, M., Bento, J. P., Almeida, P. C., Sousa, S. G., \& Ecuvillon, A., 2005, A\&A A, 437, 1127

Sousa, S. G., Santos, N. C., Israelian, G., Mayor, M., \& Monteiro, M. J. P. F. G. 2007, A\&̊A, 469,783

Sousa, S. G., Santos, N. C., Mayor, M., Udry, S., Casagrande, L., Israelian, G., Pepe, F., Queloz, D., \& Monteiro, M. J. P. F. G. 2008, A $\& A$ A, 487, 373

Sneden, C. 1973 Ph.D Thesis, University of Texas.

Takeda, Y. 2007, PASJ, 59, 335 